\title{
"Dépoussiérer" des techniques anciennes pour stimuler l'observation sensorielle dans la pratique du terrain
}

"Dusting off" old techniques for better sensory observation in fieldwork practices

\section{Robin Larsimont}

\section{(2) OpenEdition}

Journals

Édition électronique

URL : http://journals.openedition.org/belgeo/10781

DOI : 10.4000/belgeo.10781

ISSN : 2294-9135

\section{Éditeur :}

National Committee of Geography of Belgium, Société Royale Belge de Géographie

\section{Édition imprimée}

Date de publication : 16 décembre 2013

ISSN : 1377-2368

\section{Référence électronique}

Robin Larsimont, «"Dépoussiérer" des techniques anciennes pour stimuler l'observation sensorielle dans la pratique du terrain », Belgeo [En ligne], 2 | 2013, mis en ligne le 16 décembre 2013, consulté le 19 avril 2019. URL : http://journals.openedition.org/belgeo/10781; DOI : 10.4000/belgeo.10781

Ce document a été généré automatiquement le 19 avril 2019.

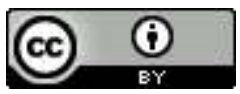

Belgeo est mis à disposition selon les termes de la licence Creative Commons Attribution 4.0 International. 


\section{"Dépoussiérer" des techniques anciennes pour stimuler l'observation sensorielle dans la pratique du terrain}

"Dusting off" old techniques for better sensory observation in fieldwork practices

\section{Robin Larsimont}

\section{Introduction}

1 Jusqu'à la première moitié du XX siècle, une des principales sources d'informations et de connaissances pour le géographe était le déplacement et le terrain (Rundstrom \& Kenzer, 1989). Au sein de diverses écoles, les pratiques de terrain de certaines personnalités allaient alors influencer de nouvelles générations de géographes. Cependant, les nouveaux paradigmes et outils quantitatifs de l'analyse spatiale de la "nouvelle géographie" feront de l'ombre à cette approche, allant jusqu'à la considérer comme anecdotique (Pedone, 2000 ; Rundstrom \& Kenzer, 1989). Parallèlement pourtant, depuis la fin des années 1970 l'intérêt de questionner le terrain refait timidement surface, tantôt sous l'égide de la géographie radicale francophone (Lacoste, 1977 ; Kayser, 1978; Giblin, 1978), tantôt sous l'influence de certaines géographes féministes (Katz, 1994 et 1996; Rose, 1997; Pedone, 2000; Sundberd, 2003). À ces remises en question, soulignant l'absence de dialogues sur les politiques du terrain et pointant le manque d'autocritique quant à l'accès et à la construction du savoir, viennent s'ajouter plusieurs contributions originales dans le cadre de colloques ou de numéros de revues consacrés au sujet ${ }^{1}$.

2 Cependant, sur base de cet éventuel retour du terrain en géographie, deux observations méritent d'être soulevées. Premièrement, force est de constater que l'étudiant en géographie reste peu préparé à l'exercice de terrain. Les lignes de force de cette pratique sont certes souvent diluées dans différents cours ou excursions. Mais le manque de 
manuels, de guides, de cours ou de revues spécialement centrés sur le terrain semble témoigner encore une certaine crainte quant à la non-professionnalisation de cette technique d'accès à l'information. Deuxièmement, il semblerait que les efforts pour comprendre les gestes de métiers des géographes de terrain s'inscrivent plus dans une exploration épistémologique de la discipline, que dans une réelle volonté de leur redonner une nouvelle vie (Wolff, 2008 et 2006; Courtot, 2006; Robic, 1988). Ces techniques seraient-elles obsolètes face aux objets et méthodes modernes d'une géographie "en adhésion à la dynamique de l'actuel" ? (George, 1990, p. 75). Demandonsnous plutôt comment construire un corpus théorique et pratique du terrain qui ne fasse pas table rase des techniques anciennes mais au contraire les expose à la lumière de certaines réflexions actuelles en sciences sociales. Cet article propose de contribuer à répondre à cette vaste question, en se munissant des réflexions qui ont accompagné la préparation au terrain, sa pratique et sa "digestion" après coup, dans le cadre d'études portant sur la gestion de l'eau en milieu rural'. Il expose donc la manière dont l'auteur s'est penché sur l'histoire et l'actualité du terrain en géographie pour alimenter sa pratique et par ce biais renforcer sa propre méthode géographique.

Dans un premier temps, nous soulignerons certains gestes de métiers sélectionnés au travers d'un travail bibliographique, en ciblant particulièrement des géographes classiques de l'école française, pour ensuite relever certaines critiques et réflexions plus actuelles sur la pratique du terrain en géographie. Dans un second temps, les deux espaces sur lesquels ont porté ces réflexions seront contextualisés en les articulant sous une même approche. Finalement, deux anciennes techniques et méthodes de terrain, à savoir la lecture du paysage et le travail d'enquête seront "dépoussiérées", pour ajouter un point d'orgue à cette réflexion d'ensemble, en rappelant leurs rôles éminemment pédagogiques dans la formation du géographe.

\section{Du terrain "muet" au terrain autocritique}

Entre le "géographe de cabinet" du XIX siècle et le géographe de laboratoire des années 1980, le géographe de l'"époque classique", pour reprendre l'expression de Paul Claval, est un homme de terrain (Broc, 2010, p. 433). Que ce géographe, également qualifié de moderne (Wolff, 2008), parcoure le terrain seul pour ses recherches ou avec ses étudiants à des fins pédagogiques, il est d'abord un grand marcheur qui acquiert sa connaissance du monde au fil des kilomètres, en partant des évidences sensibles et de l'observation du paysage (Broc, 2010, pp. 433-434; Claval, 1998, p. 41; Dresch \& George, 1966). Bien que certaines écoles allaient s'attacher à l'étude des interactions hommes-milieu, que d'autres interrogeaient la différenciation régionale ou encore allaient avoir pour objet le paysage, toutes avaient en commun ces traits : faire de la géographie une science de l'observation (Claval, 1998, p. 41; Sauer, 1956). Or avant d'apprendre à vraiment observer, le géographe, dont la discipline était encore coincée dans un coin inconfortable (Harvey, 1998) devait disposer de grilles de lecture, souvent héritées de la topographie, de la géologie ou de la botanique (Capel, 1977 ; Higueras Arnal, 2003, p. 27). Dans ce contexte, la géographie rurale - une des pièces maîtresses de la progressive consolidation de la géographie humaine (Chapuis, 1998, p. 149) - s'alimenta et s'appropria certains concepts en relations avec son principal objet d'analyse, le paysage agraire. Ce filtre conceptuel encore flou accompagnera une méthode essentiellement inductive, qui partait de l'observation de cas pour s'élever, notamment grâce à la comparaison dans l'espace et 
dans le temps, au classement et à la compréhension des phénomènes (Chapuis, 1998, p. 150).

5 Cependant, alors que l'enseignement de ce "savoir-voir" se transmettait du maître au disciple par une forme de baptême du terrain (Sundberg, 2003; Calberac, 2011), la méthode utilisée restera le plus souvent "muette" dans la production scientifique finale. En d'autres termes et comme le souligne Marie-Claire Robic "si le terrain est valorisé comme l'instance majeure de construction, de transmission et de la validation de la vérité géographique, on connaît assez mal les gestes de métier que cette foi dans le terrain implique."(Robic, 1996, in Wolff, 2008). Ce manque de transparence sur la méthode de terrain serait donc une première faiblesse essentielle de la démarche de ces géographes classiques. Mentionnons cependant l'initiative de l'américain William. M. Davis, dans le cadre d'excursions interuniversitaires au début $\mathrm{du} \mathrm{XX}^{\mathrm{e}}$ siècle. Ces dernières visaient principalement les confrontations collectives des méthodes de description et d'observation géographique sur le terrain par différents géographes. Par la question suivante que Davis posait à ses collègues issus de divers continents; "quelle est votre méthode pour faire une description du paysage que voici?" (Davis, 1912), il voulait dégager certaines lignes de force de la méthode de terrain et en souligner la pluralité. Ce dernier influencera une génération de géographes particulièrement convaincus de l'importance des données collectées sur le terrain (Rundstrom \& Kenzer, 1989), tout comme plus tard Carl Sauer avec son approche morphologique du paysage et pour qui le terrain, plus exploratoire que méthodique, reflétait la vraie matrice dans laquelle consolider une curiosité géographique (Gade, 2011, p. 176 ; Sundberg, 2003). Cependant, hormis quelques exceptions, le manque de transparence sur la méthode de terrain persistera longtemps au sein de la discipline. Il sera d'ailleurs particulièrement palpable en France, où Paul Vidal de la Blache, en généralisant cette pratique, essaya d'imposer à ses étudiants "un certain privilège de l'approche directe par le regard" (Claval, 1998, p. 101). Les géographes vidaliens, entraînés d'emblée à aller sur le terrain dans leur formation initiale (Wolff, 2008) élaboraient une thèse régionale de géographie ${ }^{3}$ au travers de laquelle ils allaient façonner à leur manière certaines recettes méthodologiques de terrain. Effectivement, malgré l'héritage commun de ce goût pour une observation qui porte "surtout sur des réalités de plein air immédiatement visibles pour le chercheur" (Claval, 2007, p. 42), chacun personnalisera sa démarche, en privilégiant certains outils ou techniques. Ainsi, si l'observation et le questionnement du paysage depuis les points de vues élevés, permettant une lecture globale, semble une entrée privilégiée et commune, chacun cherchera l'élément qui, dans l'ensemble, lui permettra d'interpréter et dans certains cas d'atténuer à sa manière le concept lourd de "genre de vie", hérité de Vidal.

6 L'étape préliminaire de la préparation au terrain a donc visé à sélectionner certains thèmes ou techniques d'observation que la lecture de textes laissait entrevoir. Par ailleurs, afin de comprendre la relation qu'entretenaient certains de ces géographes avec le terrain et la manière d'obtenir et de transcrire l'information qu'ils y puisaient, l'usage de contributions, partant de carnets de notes, de correspondances ou d'écrits a facilité la sélection de certains gestes de métiers (Wolff, 2006 et 2008; Courtot, 2006; Broc, 2010 ; Calberac, 2011). En effet, en se plongeant, au travers de ces différentes sources, dans cette question des rapports au terrain des géographes et en la situant dans l'histoire longue de la construction des savoirs (Wolff, 2008), on découvre des procédés et des techniques qui peuvent se révéler fort utiles. Distinguons donc particulièrement parmi ces géographes vidaliens certains objets et méthodes attachés à quelques personnalités de la géographie. 
Par exemple, dans la thèse que Jean Brunhes consacre à l'irrigation en Méditerranée il défend que cette dernière, "en pays aride est l'œuvre qui par excellence marque en traits saisissants sur la surface de la terre les résultats de l'activité persévérante de notre espèce" (Brunhes, 1902, p. 425, in Robic, 1988). À côté de ce principal filtre d'interprétation des relations hommes-milieu, Brunhes portera aussi un regard quasi ethnologique sur l'habitat. Cependant, la principale figure de cet objet d'analyse est Albert Demangeon (1926) qui, se munissant d'une méthode quasi anatomique, considérait l'exploitation rurale comme une cellule vivante dont l'habitation serait le noyau (Gottmann, 1947). Cet intérêt pour l'habitat rural sera plus tard également une des pierres angulaires de Pierre Deffontaines, dans son souci de comprendre la genèse des paysages et au travers de sa sensibilité d'artiste à l'égard de ceux-ci (Claval, 1998, p. 204). Par ailleurs, Max Sorre (1913), malgré son approche plus naturaliste, s'y penchera également, tout comme Maurice Le Lannou dans son questionnement de l'homme-habitant.

7 Certains de ces géographes, pour passer d'un regard "passif" à un regard "actif" lors de l'exercice d'observation, avaient recours aux croquis d'éléments du paysage ou de son ensemble, afin de transformer celui-ci en objet de connaissance (Giblin, 1978). Ces croquis souvent sans complexe et grand souci esthétique pour Vidal (Courtot, 2006) mais au contraire pointilleux chez De Martonne, et plus tard chez Deffontaines ou Jean Dresch, "ouvrent les yeux du dessinateur" sur beaucoup de détails qui échapperaient au simple regard (Broc, 2010, p. 242 ; Bataillon, 1999, p. 72). Le dessin est donc un moyen, au même titre que la note écrite, d'enregistrer une réalité géographique observée (Courtot, 2006) tout en étant déjà une interprétation de celle-ci (Broc, 2010, p. 242). La photographie quant à elle, outil d'abord réservé à un cercle relativement restreint de praticiens, deviendra également un outil précieux pour l'homme de terrain. Bien qu'utilisée presque systématiquement par de Martonne (Broc, 2010, p. 243) ou par Demangeon (Wolff, 2006), on attribue le plus souvent cette méthode "iconographique" au "géo-photographe" Jean Brunhes, dont la photographie était une des bases de son enseignement assisté par l'image (Jean-Brunhes Delemarre et al., 1993, p. 60). Vidal rappelait d'ailleurs, en se référant à cette technique moderne, qu'il fallait "qu'elle soit empruntée dans un esprit géographique, par des personnes sachant épier la nature" (Vidal, in Jean-Brunhes Delemarre \& al.1993:60). D'autre part, nombreux étaient ceux qui combinaient l'usage de la photographie au dessin (Robic, $1993: 122$ ), une manière d'accompagner une "vue raisonnée" ou une "description explicative" de la surface de la terre, essence même de leur géographie. C'est d'ailleurs à la même époque et plus particulièrement pendant la Première Guerre mondiale que l'on découvrit l'intérêt que présentaient la vision et la photographie aérienne pour contempler, sans la remplacer, la vision traditionnelle de la terre (Broc, 2010, p. 438). Emmanuel de Martonne qui sera l'un des premiers en France à comprendre le caractère irremplaçable de cet instrument en géographie (Broc, 2010, p. 433) souligne notamment qu'elle permet “à la fois la perception des détails et celle des ensembles, l'analyse et la synthèse" (De Martonne, in Broc, 2010, p. 440).

Cette géographie ne pouvait cependant pas être fondée exclusivement sur l'observation du paysage mais au contraire devait s'intéresser à ceux qui habitaient les espaces sur lesquels elle se penchait et à la manière dont ces derniers s'inséraient dans le milieu, l'exploitaient et le modelaient (Claval, 2007, p. 44). Max Sorre défendait par exemple, que "l'endroit où l'on apprenait le plus en géographie humaine était la place du village où l'on s'assoit sur un banc [...]" (Bataillon, 1999, p. 107). Ce travail d'observation impliquait donc d'aller à la rencontre des habitants et de discuter avec tous ceux qui pouvaient faciliter la 
compréhension de l'espace étudié, aussi bien les maîtres d'école, que les ouvriers ou les paysans (Wolff, 2006; Chapuis, 1998, p. 150). Néanmoins, bien qu'il soit important de mentionner par exemple l'effort d'Albert Demangeon dans l'élaboration de questionnaires sur l'habitat rural (1926), ces incursions vers des faits de micro-échelle restaient limitées, car elles n'avaient d'autre motif que d'éclairer ce qui était visible (Claval, 2007, p. 43). Soulignons donc ici une deuxième faiblesse de cette démarche de géographie classique, à savoir la foi en un terrain, "admis comme le concret et non comme une représentation que l'on pourrait en avoir" (Labussière \& Aldhuy, 2008). La contestation de cette forme d'objectivation basée sur l'évidence des objets du terrain et l'occultation de l'action du sujet (Labussière \& Aldhuy, 2012) ne se consolidera réellement qu'à partir des années 1970 puis 1980 sous l'influence de la géographie humaniste, structuraliste et poststructuraliste (Rhoads \& Wilson, 2010, p.32). La conception positiviste de l'observation sera dès lors systématiquement défiée.

Par exemple en France c'est à la revue Hérodote et à son effort pour refonder la discipline, que l'on doit une réelle introspection de l'exercice et des politiques du terrain. Tout en questionnant l'autorité de l'observation "vue d'en haut" du géographe et en s'ouvrant à la sociologie et à l'ethnologie, deux numéros intitulés L'enquête et le terrain (Hérodote, 1977 et 1978) abordent aussi bien la relation d'enquête, l'analyse paysagère que les excursions. Par ailleurs et particulièrement dans le monde anglo-saxon, soulignons le rôle qu'ont joué les critiques des géographes féministes dans la remise en question générale du terrain en géographie. En se centrant sur la production des savoirs géographiques et la manière dont le masculinisme obscurci ses conditions d'accès (Rose, 1997 ; Sundberg, 2003) elles pointent du doigt l'aspect neutre et "dépersonnifié" du chercheur. L'autorité du regard du géographe sur la relation entre l'homme et la terre, depuis sa colline (Sundberg, 2003), doit maintenant passer par le filtre du genre, de la position sociale, de la race, des intérêts politiques et personnels historiquement construits de l'observateur (Sundberd, 2003; Katz, 1994 ; Rose, 1997 ; Pedone, 2000). Après le "Voyez et pensez!" que Davis disait autrefois aux jeunes géographes en quête d'espaces inexplorés (1912) la place est donc au "Voyez et pensez à vous en train de voir !".

Aussi, Cindi Katz, dans sa volonté de contextualiser complètement le travail de terrain, ouvre ces frontières, en considérant que le chercheur, au cours des déplacements dans sa pratique, se situe dans des "espaces intermédiaires" (Katz, 1994). C'est-à-dire qu'il n'est ni totalement dans la zone d'étude ni totalement en dehors, et que la construction de sa connaissance est un processus social imbriqué dans des réseaux de relations de pouvoir (Katz, 1994). Après ce terrain, considéré comme un des espaces-temps dans lequel une connaissance se construit (Germes, 2008), le chercheur est donc appelé à davantage exposer le contexte de production des données, à la fois théorique et pratique (Petit, 2010). Au sein de cette transparence requise, une autobiographie critique est du coup indéniable, tout en veillant cependant à ne pas convertir notre conscience personnelle en place centrale de l'étude. Par ailleurs, mentionnons qu'un certain relativisme méthodologique s'est consolidé sous l'influence poststructuraliste, en avançant par exemple l'opération de déconstruction comme méthode de décryptage du réel (Dixon, 2010 ; Rhoads \& Wilson, 2010 ; Robbins, 2010). Cette approche critique, en examinant les prétentions au savoir, particulièrement celles de sources scientifiques a le mérite de reconsidérer quasi généalogiquement (Robbins, 2010, p. 250) certains concepts considérés comme acquis au sein des appareils descriptifs et explicatifs de la géographie. Autrement 
dit, une certaine distance critique vis-à-vis du bagage conceptuel du chercheur semble requise avant et pendant son terrain.

\section{L'observation comme outil "tout-terrain"}

11 Cette réflexion sur le travail de terrain a pour toile de fond deux expériences dans lesquelles l'auteur s'est penché sur des problématiques socio-environnementales liées à l'eau et aux relations de pouvoir que sa gestion incarne en milieu rural aride. L'observation et le questionnement de paysages de l'eau (superficielle et souterraine) dans leur complexité a servi de prisme pour analyser certains conflits environnementaux sous-jacents.

Avant de contextualiser les cas d'études et de diagnostiquer l'exercice de terrain en le considérant comme une pratique de l'observation directe (Higueras Arnal, 2003, p. 267), quelques éclaircissements sur deux autres modalités de l'observation sont nécessaires. Premièrement, l'observation indirecte ou instrumentale, faisant référence à l'utilisation de techniques et instruments permettant de "percevoir les systèmes territoriaux et les ensembles spécifiques d'objets d'intérêt géographique dans toute leur complexité et distribution territoriale" (Higueras Arnal, 2003, pp. 271-272). Il s'agit principalement des outils cartographiques (cartes topographiques, SIG, télédétection,...). Deuxièmement l'observation documentaire, à savoir "l'ensemble de données se référant à des faits actuels ou historiques aussi bien statistiques que narratifs" (Higueras Arnal, 2003, p. 287). Une partie de cette information s'obtient directement sur le terrain en parcourant les bibliothèques locales, les archives, mais aussi au travers de la littérature grise. Ces deux types d'observation, en permettant au chercheur une contextualisation spatio-temporelle de sa zone d'étude et en renforçant ses connaissances sur la genèse et l'histoire locale des paysages observés, encadreront et rétro-alimenteront sans cesse son exercice d'observation directe.

\section{Deux waterscapes : quand l'eau incarne une multitude}

La construction du contexte de la zone d'étude qui accompagnera l'investigateur dans sa pratique d'observation directe dépend de sa "manière de voir les choses", et comment celle-ci a pu évoluer sous l'influence de certains paradigmes. Soulignons donc brièvement certaines influences qui ont dicté la lecture de ces paysages de l'eau. Tout d'abord, un intérêt pour le recours à certaines métaphores afin de décrire l'épaisseur historique du paysage. Citons par exemple, celle du palimpseste où transparaissent les effets des techniques oubliées (Gourou, 1973, p. 31 ; Santos, 2000, p. 87), ou l'analyse stratigraphique visant à distinguer des "couches sédimentaires" (Renard, 2002, p. 11). Ensuite, l'idée que l'aspect quelque peu figé de cette méthode stratigraphique pouvait s'estomper en considérant ces strates comme l'origine possible de recyclages et d'inerties, c'est-à-dire des forces "qui ont en commun d'introduire du passé dans le déroulement actuel des processus" (Baudelle \& Regnauld, 2004, p.61). Enfin, cette volonté de dynamiser la trilogie eau-paysage-pouvoir, ne pouvait que rebondir sur certains concepts et outils analytiques empruntés à la Political Ecology 4 , "nouveau" convoi de la géographie anglosaxonne. Effectivement, cette approche, soucieuse de ne pas considérer les paysages comme des représentations statiques du pouvoir (Neumann, 2011) a alimenté de nombreux concepts inspirés de "termes fluides" (Linton, 2010, p. 24). Celui de waterscape, 
par exemple, au cœur de la réflexion d'Erik Swyngedouw, souligne le caractère hybride d'un paysage de l'eau, en partie naturel, en partie social, incarnant une multitude de relations et processus historico-géographiques (Swyngedouw, 1999; 2004, p. 2). Cette notion est utilisée pour comprendre les interactions entre les processus physiques, les pratiques matérielles, culturelles et les constructions idéologiques de la valeur de l'eau (Blanchon \& Graefe, 2012; Molle 2012). En incarnant l'expression des interactions entre les sociétés et leur environnement, ces waterscapes sont considérés comme des arènes aux limites flexibles dans lesquelles circulent l'eau et le pouvoir (Molle, 2012; Swyngedouw, 1999) et dans lesquelles construire un problème d'investigation sur l'eau.

Étant donné qu'il s'agit avant tout dans cet article de nous centrer sur l'exercice de terrain et non pas sur les problématiques socio-environnementales qui caractérisent ces espaces, nous n'allons donc pas nous attarder sur leurs enjeux. Néanmoins, une certaine contextualisation mérite d'être faite, et cela de façon imbriquée étant donné les fortes ressemblances des deux cas d'étude.

Tout d'abord, soulignons que l'origine étymologique du nom de ces deux régions annonce le caractère aride de celles-ci. Alors que Cuyo, nom de la région à laquelle appartiennent les piedmonts mendocins signifie "sables", la Mancha de l'arabe Manxa signifie "Terre sans eau". Avec respectivement $200 \mathrm{~mm}$ de précipitation pour le bassin du rio Mendoza et $400 \mathrm{~mm}$ pour celui du haut Guadiana, l'aménagement de l'eau, outre son caractère structurant au sein de ces territoires, incarnerait également l'inertie et dans certains cas le recyclage de rapports de forces anciens. Les processus de (re)conquêtes tout d'abord, détermineront sur les temps longs les relations de pouvoir quant à la terre et à l'eau. D'un côté la reconquête du sud de la meseta espagnole, un territoire marqué par une lente agrarisation depuis l'époque romaine et renforcée par le développement de l'agriculture et de l'irrigation à l'époque arabe. De l'autre, l'appropriation par les Espagnols de petits îlots irrigués par un système de canaux d'origines huarpes, une des nombreuses civilisations hydro-agricoles, alors sous tutelle et domination de l'Empire Inca. Au XIX ${ }^{\mathrm{e}}$ siècle, ni la désamortisation ${ }^{6}$ en Espagne, ni l'indépendance de la République Argentine ne parviendront à déraciner totalement la société d'ordres et de privilèges qui s'était établie sur ces territoires. Le désenclavement de ces derniers avec l'arrivée du chemin de fer, sera l'occasion de réorganiser leurs espaces productifs et de s'intégrer dans le marché mondial. Dans les deux cas, la transformation se fera principalement au profit d'une spécialisation régionale généralisée autour de la vitiviniculture, nouveau symbole de progrès économique et social. Ce développement agricole s'accompagna à Mendoza d'une expansion du réseau de distribution superficiel et d'une progressive "appropriation gouvernementalisée" de l'eau et de la terre en faveur d'une élite régionale (Martin, 2010). Quant à la Mancha, comme une grande partie du territoire espagnol, elle portera les traces de l'hydro-politique du régime dictatorial de Paco $\mathrm{rana}^{7}$ et du maintien garanti sur le long terme de la stabilité du système latifundiste ébranlé lors de la guerre civile (Swyngedouw, 2007). Finalement et conjointement à la restructuration globale du système agroalimentaire depuis les années 1980, la globalisation de la consommation standardisée du vin (Harvey, 2007, p. 424) imposera de nouvelles transformations spatiales. Celles-ci viendront se superposer à ces territoires historiquement construits en relation à l'eau, avec parmi les conséquences de lourds impacts environnementaux. Aux habituels facteurs attribués à la surexploitation d'importants aquifères ${ }^{8}$ de la Mancha viennent s'ajouter le rôle de certains "aguatenientes" ou latifundistes de regadío enracinés pour une partie dans l'histoire de la noblesse et bourgeoisie locale (Ruiz, 2008; 
Swyngedouw, 2007). Du côté de Mendoza, une nouvelle dynamique territoriale se développe avec la conquête des piedmonts par l'agrobusiness', à partir de l'exploitation des réserves souterraines d'eau, principalement en amonts du réseau d'irrigation superficiel traditionnel (Montaña, 2012, p. 69, Larsimont, 2012).

\section{L'observation directe ou sensorielle}

ment approcher l'interconnectivité à la fois hydrologique et socio-politique qu'incarnent les circulations d'eau et de pouvoir au sein de ces waterscapes? Dans la plupart des cas ces flux passent au travers des mailles des analyses quantitatives et, comme le rappelle Paul Robbins (2010, p. 244), le travail de terrain est souvent essentiel pour leur donner un sens et produire des explications fiables sur la manière dont ils fonctionnent et mieux apprécier leurs effets. À ce propos, nous soutenons cependant que dans l'approche positiviste de l'observation de certains géographes classiques, tout n'est pas à jeter. Au contraire, que le recours à certaines techniques anciennes du terrain, comme la lecture du paysage combinée au travail d'enquête, reste une entrée possible dans l'analyse de la complexité de l'espace étudié. Ceci, bien entendu à condition de considérer que "chaque chercheur entre dans son terrain avec un dispositif propre de mise en visibilité de la réalité" et que les résultats de sa recherche se basent sur un terrain vécu, observé, interprété (Petit, 2010). Penchons-nous donc sur deux modalités de l'observation directe -également qualifiée de sensorielle- (Higueras Arnal, 2003, p. 267), inspirées de techniques anciennes mais rajeunies par certaines influences nouvelles.

\section{La lecture du paysage et le travail d'enquête}

Si l'on se centre sur le regard, l'observation directe peut s'entendre comme une manière de voir ou plutôt comme l'affirmait Pierre Gourou (1973) une "mise en question permanente de ce qui se voit". Rappelons que le paysage occupait une place centrale dans sa géographie, dont le principal souci était "d'expliquer pourquoi les choses sont telles qu'on les voit" [...] et s'efforcer "de dégager les facteurs responsables de ces situations ${ }^{10}$ [...]". Cette mise en accusation du paysage demande donc une curiosité sans limite que l'on retrouve également chez Carl Sauer (Sauer, 1956; Mitchell, 2000, p. 223 ; Gade, 2011, p. 75). En lecteur attentif - et tout en apprenant des gestes et habitudes des agriculteurs, des techniciens de l'irrigation, des travailleurs saisonniers ou des ingénieurs agronomes que je croisais et pouvais interroger sur mon chemin -, j'ai donc pris soin d'observer dans le détail et dans l'ensemble ces waterscapes, une carte topographique et des images satellites à la main, afin d'identifier les lignes de forces qui les caractérisaient ${ }^{11}$.

Cependant, si la lecture du paysage permet d'approcher certaines relations de pouvoir dans leurs dimensions matérielles, le travail d'enquête lors du terrain la complète en se penchant sur les pratiques discursives, idéologiques et culturelles. L'empirisme de cette méthode, avant tout tributaire des équipements cognitifs de l'enquêteur et des dispositifs techniques et théoriques sur lesquels il s'appuie (Céfaï, 2003, p. 197), consiste en l'exercice d'une attention soutenue pour considérer un ensemble circonscrit de faits, d'objets, de pratiques (Arborio \& Fournier, 2010, p. 9). Sans pour autant avoir recours à l'exploration intense que fournit "l'observation participante" à proprement parler, la relation d'enquête s'est réalisée au travers de diverses techniques issues de ce que Paul Robbins qualifie "d'approches d'appréciation participative" (Robbins, 2010, p. 246). Ces techniques s'apparentent à certaines recommandations de Bernard Kayser quant à l'étude des 
sociétés rurales, dont "la dynamique sociale [serait] révélée par les conflits" et dont il défend que l'identification de ceux-ci "est la première tâche de l'enquêteur" (Kayser, 1978). Ce dernier prône alors de s'imprégner, moyennant une analyse historique préalable, de l'atmosphère sociale et de déceler dans les conversations banales les signes de tensions profondes (1978) notamment avec le recours à l'entretient d'imprégnation ou approfondi (Kayser, 1989, p. 84). À ce propos, comme l'illustre le témoignage suivant sur les conditions de travail des enfants dans les oasis de Mendoza, le maitre d'école peut encore s'avérer une personne source, particulièrement dans les petites localités rurales.

"J'ai des élèves de 13 ans à l'école de Lavalle, qui me brisent le cœur quand ils me racontent que chaque été ils font les récoltes, bien sûr au noir et que certains se blessent accidentellement. Bien qu'ici à Mendoza les contrôles deviennent timidement plus fréquents, trop souvent encore les entrepreneurs, avant l'arrivée des inspecteurs (qui les appellent préalablement) les envoient se cacher. Ce sont des réalités sociales que malheureusement nous sommes encore loin d'enterrer" (Témoignage d'un enseignant d'une école du département de Lavalle (Mendoza) enregistré en juillet 2010).

D'autre part, qui dit tensions et conflits souligne bien souvent aussi l'atmosphère de suspicion qui peut régner autour de l'enquêteur. En effet, réaliser une étude qui aborde la surexploitation des écosystèmes aquatiques sur un territoire sujet à de nombreuses fraudes, demande certaines précautions vis-à-vis des personnes enquêtées. Comme le souligne Cindi Katz (1996, p. 173), à partir de son expérience au Soudan, ceci implique de se pencher sur certains choix stratégiques dans sa pratique de terrain et sur l'impact que ceux-ci peuvent avoir sur le contenu de la recherche. Parmi ces choix stratégiques figure la possibilité lors de l'entrée sur le terrain et la prise de contact de s'appuyer sur ce que Bernard Kayser appelle un "informateur privilégié" (Kayser, 1989, p. 90). Une distance critique vis-à-vis de l'information facilitée par ce "guide bienveillant au premier abord" est cependant indispensable.

\section{La construction subjective d'un paysage}

Après ce travail d'observation directe, s'en suivit une observation raisonnée, autrement dit, la construction subjective d'une synthèse de l'espace observé. Cette dernière, construite au sein de ces "espaces intermédiaires" caractérisés par Cindi Katz (1994), a pris entre autres la forme d'une synthèse graphique. L'élaboration de celle-ci, s'est réalisée en combinant la prise de notes, de photographies, de croquis paysagistes ou cartographiques sur le terrain et certains repères spatiaux qu'offraient les outils d'observation indirecte. La technique utilisée pour retranscrire mes observations n'a donc pas été la description de paysages vus, mais au contraire, le recours à la construction de différents paysages-types. Cet exercice graphique fait en quelque sorte écho aux paysages vidaliens, qui se référaient à un décor construit, à des paysages créés à partir de synthèses régionales (Giblin, 1978; Claval, 2007, p.64). La construction de cette représentation abstraite de l'espace (Lacoste, 1990, pp. 32-33) aboutit à un paysage non localisé précisément, mais qui, par une série de sélections et de montages serait reconnaissable quel que soit le lieu dans une aire régionale et quelle qu'en soit la saison (Giblin, 1978). D'autre part, étant donné que l'interprétation et l'analyse du paysage sont difficilement séparables de la fabrication de la carte (Bataillon, 1999, p.70), cette observation raisonnée a impliqué également la construction d'une synthèse cartographique (figure 1). 
Figure 1. Synthèse cartographique des unités de paysages : élaboration personnelle.

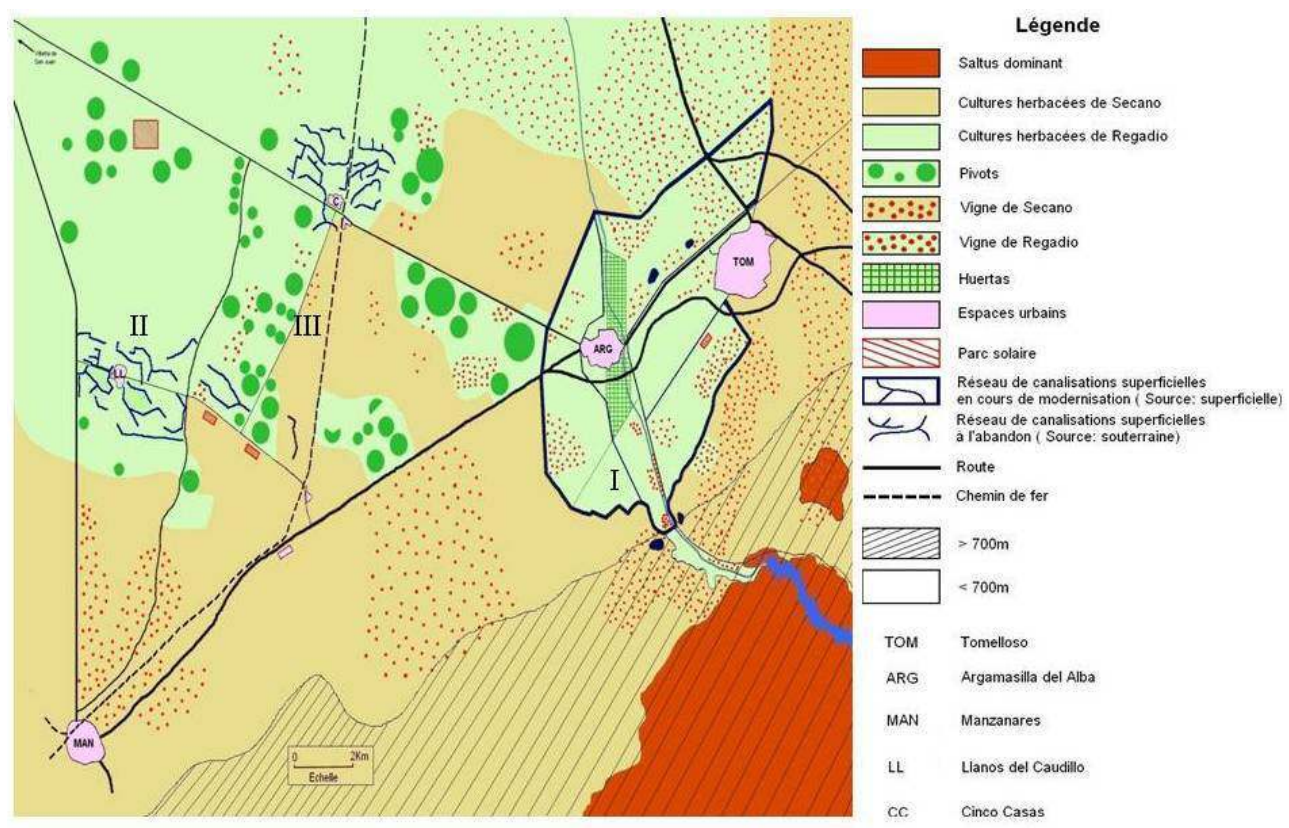

Dans le cas de l'étude sur la Mancha, cette technique a débouché sur la distinction de diverses unités de paysage au travers desquelles questionner les modalités d'accès et de distribution de l'eau. Une première unité, portant les traces des travaux hydrauliques de l'époque franquiste consistait en un périmètre d'irrigation alimenté par un barrage, localisé sur les hauteurs (figure 2). Un réseau de canalisations superficielles situé en aval de ce dernier imposait une distribution tour à tour de l'eau, contrôlée jusque très récemment par une police des eaux, mais aujourd'hui automatisée. La deuxième unité met en évidence les villages de colonisation, autres vestiges de la mission hydraulique franquiste (figure 3). Ces villages compacts, isolés au milieu d'un espace dominé par la grande propriété, sont entourés d'un périmètre d'irrigation par canalisations superficielles. Ce dernier était alimenté, avant la déclaration de surexploitation des aquifères, par une batterie de puits collectifs, qui distribuait l'eau sur les différentes parcelles des colons, d'une dizaine d'hectares chacune. Par ailleurs, à côté du rôle de vitrine de la propagande agraire du régime, comme en témoigne le nombre d'écussons de l'Instituto Nacional de Colonización (INC) que l'on retrouve sur les ruines de ces infrastructures, ces villages servaient également de réserve de main-d'œuvre pour les grandes exploitations des environs. On ne peut que douter de la réelle transcendance économique et sociale de ces enclaves planifiées exclusivement sur l'agriculture, quand on observe l'actuel abandon des infrastructures hydrauliques collectives. Seuls certains colons ont pu difficilement maintenir leur activité productive à partir de puits individuels. La troisième unité, finalement, représente les grandes propriétés des alentours, le plus souvent sous forme de sociétés anonymes (figure 4). Munies pour la plupart de plusieurs pivots d'irrigation, ces exploitations témoignaient d'une gestion plus entrepreneuriale, combinant la vitiviniculture et l'alternance de cultures selon les conjonctures (maïs, luzerne, alliums,...). 
Figure 2. Paysage-type (I) du périmètre d'irrigation alimenté depuis un barrage. Elaboration personnelle.

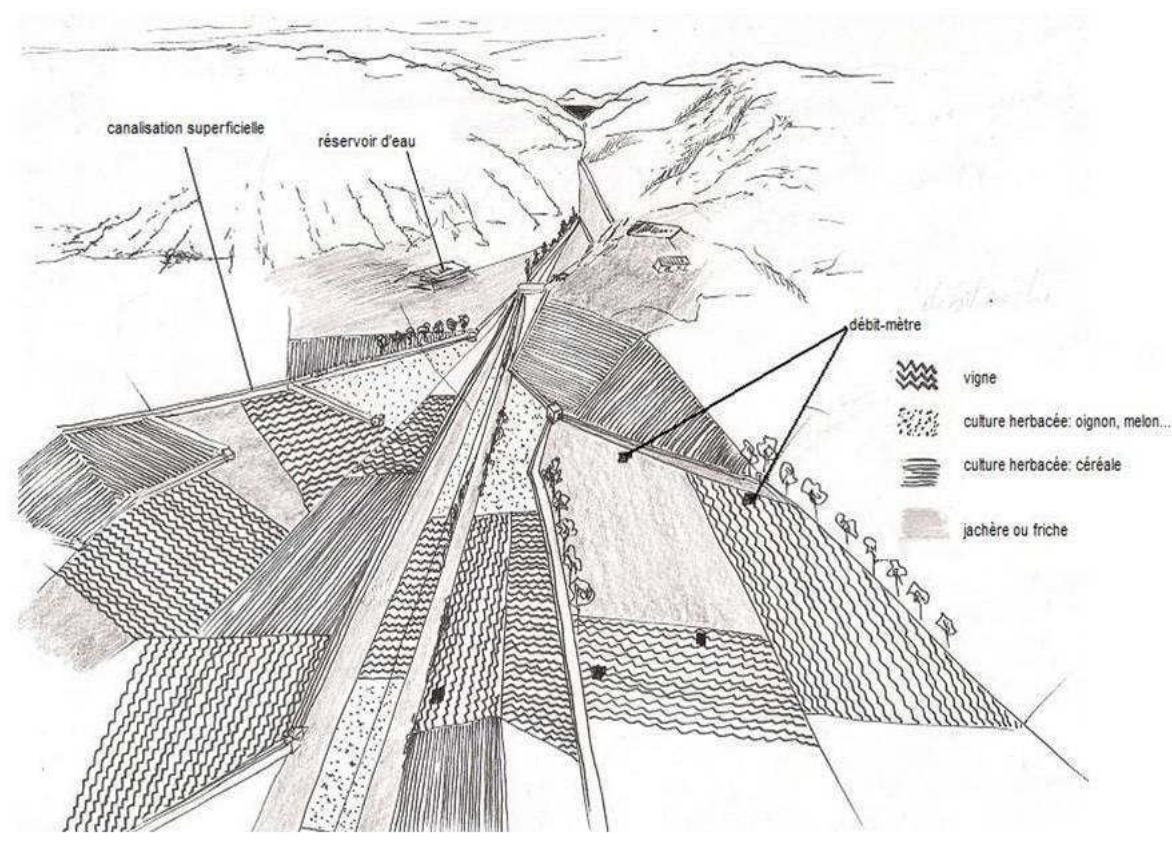

Figure 3. Paysage-type (II) des villages de colonisation de l'époque franquiste. Elaboration personnelle.

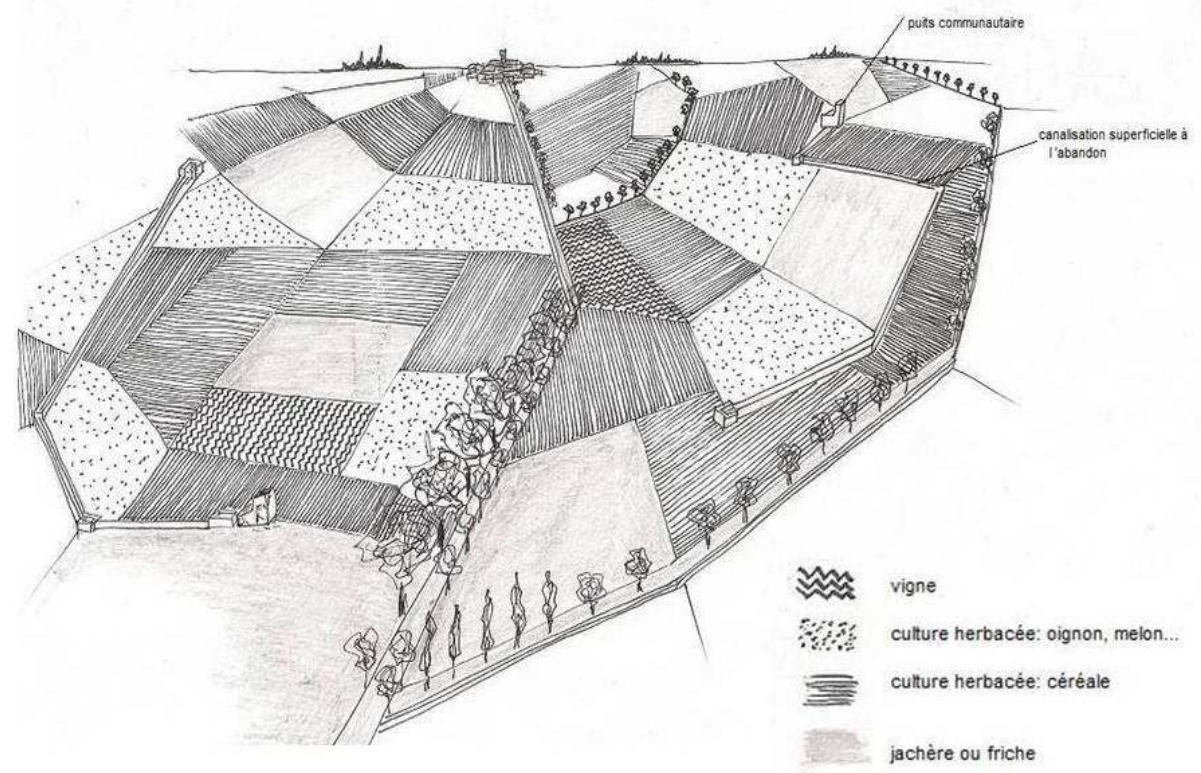


Figure 4. Paysage-type (III) des espaces dominés par les pivots d'irrigation. Elaboration personnelle.

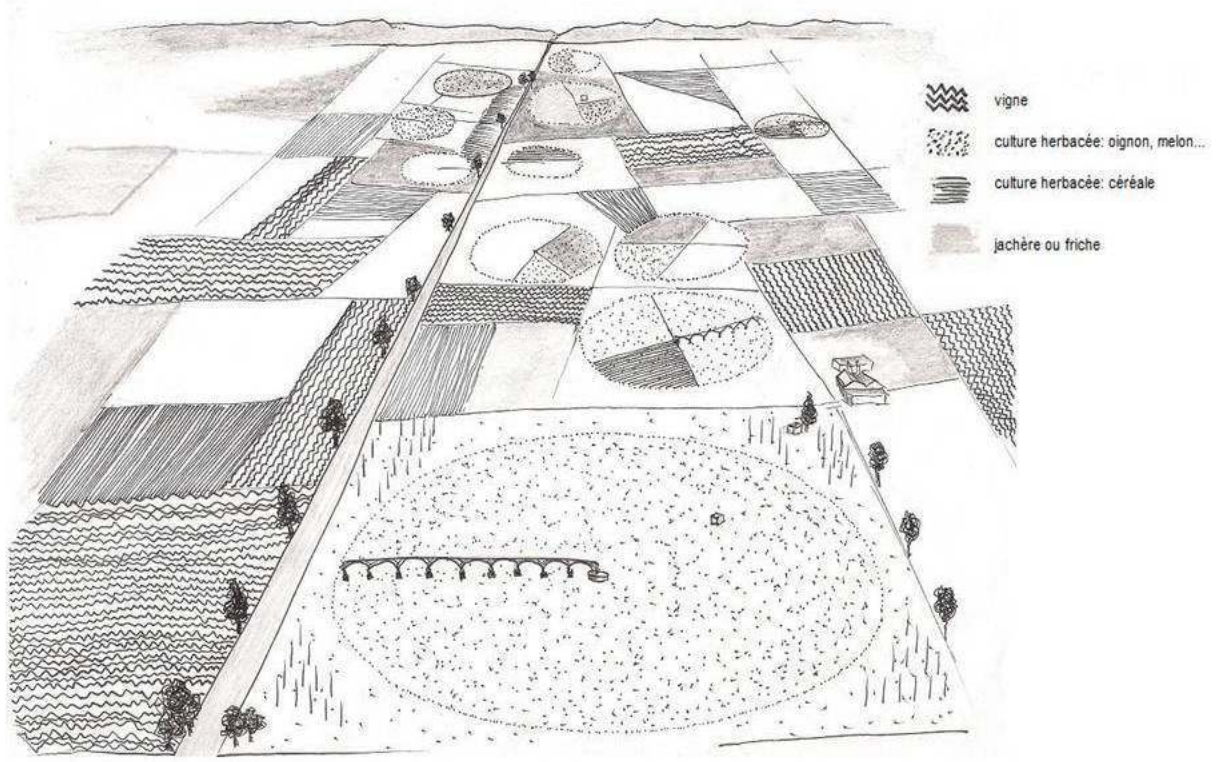

Dans le cas d'étude de Mendoza, la réalisation de la synthèse graphique (figure 5) visait à mettre en évidence, le phénomène d'expansion de la frontière agricole sur les piedmonts généré par l'agribusiness. Et comment ces entreprises, en exploitant les aquifères parviennent à développer leur activité indépendamment du système de gestion du réseau de distribution superficiel traditionnel, alimenté quant à lui par la régulation du Rio Mendoza.

Figure 5. Types d'irrigation selon la source : élaboration personnelle.

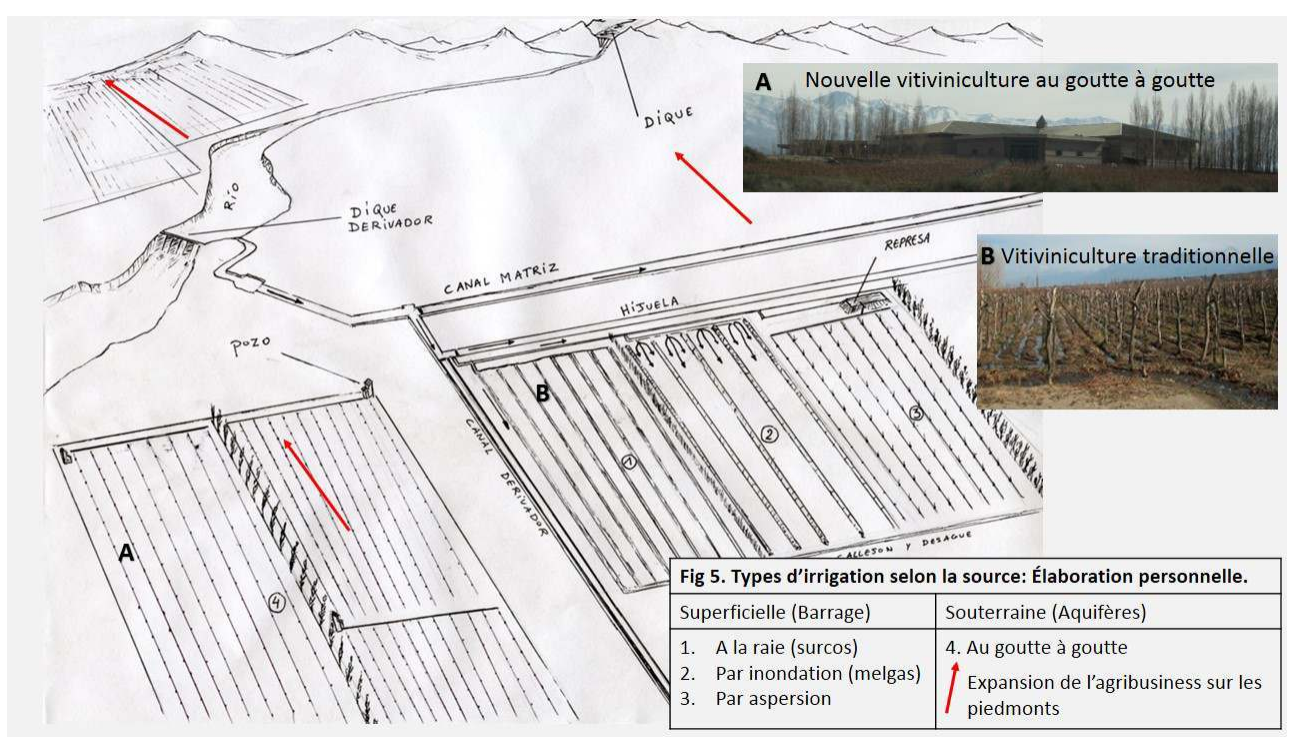




\section{Conclusion}

Le travail de terrain semble préparer son retour en géographie, comme en témoignent les contributions variées sur le sujet ces dernières années. En décryptant le terrain sous diverses questions et en l'enrichissant d'influences pluridisciplinaires, ces critiques constructives semblent cependant garder leurs distances vis-à-vis des recettes anciennes. Bien que certaines incursions biographiques dans l'histoire du terrain en géographie ont eu le mérite de faire remonter à la surface certains gestes de métiers, ceux-ci, souvent présentés sur un ton nostalgique, peuvent continuer à paraître désuets à côté des outils modernes dont dispose actuellement la discipline.

Pourtant, comme l'a ciblé cette réflexion, parcourir son terrain au XXI ${ }^{\mathrm{e}}$ siècle à la manière d'un géographe du début du siècle passé, en prenant compte de la subjectivité de cette entreprise, permet de prendre conscience de dimensions trop souvent cachées derrière les raccourcis qu'offre la technologie moderne.

D'autre part, comme il a été illustré au travers de la lecture du paysage, construire son "paysage-terrain", c'est non seulement fabriquer un outil utile pour apprendre à "savoirpenser l'espace" (Giblin, 1978) mais cela revient également à consolider sa propre méthode géographique. Par ailleurs, le travail d'enquête au contact des différents acteurs qui modèlent ces paysages, permet de transcender et de "fluidifier" leurs aspects matériels en puisant dans les dimensions discursives ou conflictuelles qu'ils incarnent. En se replongeant dans deux expériences de terrain, cette réflexion est avant tout un appel à reconsidérer la place du terrain en géographie et dans le cursus universitaire à venir.

\section{BIBLIOGRAPHIE}

ARBORIO A.M., FOURNIER P. (2008), L'observation directe, Paris, Armand Colin, 127 p.

BAUDELLE G., REGNAULD H. (2004), Échelles et temporalités en géographie, Paris, Sedes, 175 p.

BATAILLON C. (1999), Pour la géographie, Paris, Flammarion, 161 p.

BENJAMINSEN T. \& SVARSTAD H. (2009), “Qu'est-ce que la 'political ecology' ?” Natures Sciences Sociétés, 17, pp. 3-11.

BLANCHON D. \& GRAEFE O. (2012), “La radical political ecology de l'eau à Khartoum. Une approche théorique au-delà de l'étude de cas”, L'Espace géographique, 41, pp. 35-50.

BROC N. (2010), Une histoire de la géographie physique en France (XIX'e-XX ${ }^{e}$ siècle) : Les hommes - les oeuvres- les idées, Presses Universitaires de Perpignan, 716 p.

CALBERAC Y. (2011). "Le terrain des géographes est-il un terrain géographique ? Le terrain d'un épistémologue”, Carnets de géographes, 2.

CEFAÏ D. (2003), “L'enquête de terrain”, textes réunis, présentés et commentés, Paris, La Découverte, $624 \mathrm{p}$. 
CAPEL H. (1977), "Institucionalización de la geografía y estrategias de la comunidad científica de los geógrafos”, Geo Crítica, 8-9, Universidad de Barcelona, pp. 1-58.

CHAPUIS R. (1998), “La géographie agraire et la géographie rurale”, in BAILLY et al., Les concepts de la géographie humaine, Paris, Armand Colin, pp. 149-164.

COURTOT R. (2006), “Un voyage de Paul Vidal de la Blache en Espagne dans la huerta de Valence (1906)”, Cybergeo. European Journal of Geography [en ligne], $\mathrm{n}^{\circ} 354$.

COLLIGNON B. \& RETAILLÉ D. (dir.) (2010), “Le Terrain”, L’Information Géographique, 74, 1.

CLAVAL P. (1998). Histoire de la géographie française de 1870 à nos jours, Paris, Nathan Université, $543 \mathrm{p}$.

CLAVAL P. (2007), Épistémologie de la géographie, $2^{\mathrm{ème}}$ édition, Paris, Armand Colin, p. 304.

DAVIS W.M. (1912), “L'esprit explicatif dans la géographie moderne”, Annales de Géographie, 21, 115, pp. 1-19.

DeLYSER D. \& STARRS P. (coord.) (2001), “Doing fieldwork”, Geographical Review, 91, pp. 1-2.

DEMANGEON A. (1926). "Un questionnaire sur l'habitat rural”, Annales de Géographie, 35, 196, pp. 289-292.

DIXON D. (2010). “Analyzing Meaning”. in GOMEZ B. \& PAUL JONES J. (eds.), Research Methods in Geography: A Critical Introduction, Wiley-Blackwell, pp. 392-407.

DRESCH J. \& GEORGE P. (1966), “Raoul Blanchard (1877-1965)”, Annales de Géographie, 75, 407, pp. 1-5.

GADE D.W. (2011), Curiosity, Inquiry, and the Geographical Imagination, New York, Peter Lang Publishing, $308 \mathrm{p}$.

GEORGE P. (1990), Le métier de géographe : Un demi-siècle de géographie, Paris, Armand Colin, 250 p.

GERMES M. (2008), "En quête d'un 'terrain corporel', jeux de regards dans et sur la rue", Communication présentée au colloque À travers l'espace de la méthode : les dimensions du terrain en géographie, Arras, 18-20 juin 2008.

GIBLIN B. (1978), “Le paysage, le terrain et les géographes”, Hérodote, L'enquête et le terrain II, 1, pp. 74-89.

GOTTMANN J. (1947), "De la méthode d'analyse en géographie humaine”, Annales de Géographie, 56 , 301, pp. 1-12.

GOUROU P. (1973), Pour une géographie humaine, Paris, Flammarion, 388 p.

HARVEY D. (1998), “The Humboldt Connection”, Annals of the Association of American Geographers, 88,4 , pp. $723-730$.

HARVEY D. (2007), Espacios del capital. Hacia una geografía crítica, Madrid, Akal, 447 p.

HIGUERAS ARNAL A.M. (2003), Teoría y método de la geografía : Introducción al análisis geográfico regional, Zaragoza, Prensas Universitarias de Zaragoza, 447 p.

JEAN-BRUNHES DELAMARRE M., PINCHEMEL P., LESOURD M., MENDIBIL D., ROBIC M. C., SIVIGNON M. (1993), Autour du monde. Jean Brunhes, regards d'un géographe, regards de la géographie, Boulogne, Musée Albert Kahn, 347 p.

KATZ C. (1994), "Playing the field: Questions of fieldwork in geography", Professional Geographer, 46,1, pp. 67-72. 
KATZ C. (1996), “The Expeditions of Conjurors: Ethnography, Power, and Pretense”, in WOLF D.L. (ed.), Feminist Dilemmas in Field Research, Westview Press, pp. 170-184.

KAYSER B. (1978). "Sans enquête, pas de droit à la parole !", Hérodote, L'enquête et le terrain II, 1, pp. 6-18.

KAYSER B. (1989). Les sciences sociales face au monde rural, méthodes et moyens, Toulouse, Presses universitaires du Mirail, $149 \mathrm{p}$.

LABUSSIÉRE O. \& ALDHUY J. (2012), “Le terrain ? C’est ce qui résiste. Réflexion sur la portée cognitive de l'expérience sensible en géographie”, Annales de géographie, 5, pp. 687-688, pp. 583-599.

LACOSTE Y. (1977), "L'enquête et le terrain : un problème politique pour les chercheurs, les étudiants et les citoyens" Hérodote, L'enquête et le terrain I, 4, pp. 3-20.

LACOSTE Y. (1990), Paysages politiques : Braudel, Gracq, Reclus, Paris, Librairie générale française, $284 \mathrm{p}$.

LARSIMONT R. (2012), “Nuevas dinámicas del regadío y nuevos espacios rurales : el caso de la provincia de Mendoza (Argentina)", En Investigando en Rural. Actas del XVI Coloquio de Geografía Rural, Sevilla, 10-12 mayo 2012, Ulzama Ediciones, pp. 177-184.

LINTON J. (2010), What is Water? The History of a Modern Abstraction, Vancouver, UBC Press, 334 p.

MARTÍN F. (2008), “Agua y modelo productivo. Innovaciones tecnológicas e impactos territoriales en el sistema agroalimentario de Mendoza", Estudios socioterritoriales. Revista de Geografía, 7, pp. 26-45.

MITCHELL D. (2000), Cultural Geography - A Critical Introduction, Oxford, Blackwell, 325 p.

MOLLE F. (2012), "La gestion de l'eau et les apports d'une approche par la political ecology", in GAUTIER D. \& BENJAMINSEN A.T. (dir.), L'approche Political Ecology : Pouvoir, savoir, environnement, Paris, Quae, pp. 219-240.

MONTAÑA E. (2012), "Past and present vulnerabilities of agricultural and livestock producers in the Mendoza basin", in SALAS S. et al., Vulnerability to Climate Change: Challenge for adaptation in the Elqui and Mendoza basins, La Serena, pp. 55-70.

NEUMANN R.P. (2011), “Political ecology III: Theorizing landscape”, Progress in Human Geography, 35 , pp. 843-850.

PEDONE C. (2000), "El trabajo de campo y los métodos cualitativos, necesidades de nuevas reflexiones desde las geografías latinoamericas”, Scripta Nova, 57, 1.

PETIT E. (2010), “Du fil de l'eau en fils à retordre”, in COLLIGNON B. \& RETAILLÉ D. (dir.), Le Terrain, L'Information Geographique, 74, pp. 9-27.

RENARD J. (2002), La mutation des campagnes, paysages et structures agraires dans le monde, Paris, Armand Colin, $224 \mathrm{p}$.

RHOADS B. \& WILSON D. (2010), “Observing our world”, in GOMEZ B. \& PAUL JONES J. (eds.), Research Methods in Geography: A Critical Introduction, Wiley-Blackwell, pp. 26-40.

ROBBINS P. (2010), “Methods in Human Environment Geography”, in GOMEZ B. \& JONES J.P. (eds.), Research Methods in Geography: A Critical Introduction, Wiley-Blackwell, pp. 241-256.

ROBIC M.C. (1988), “Les petits mondes de l'eau. Le fluide et le fixe dans la méthode de Jean Brunhes”, L'Espace Géographique, 1, pp. 31-42. 
ROSE G. (1997), "Situating knowledges: positionality, reflexivities and other tactics", Progress in Human Geography, 21, 3, pp. 305-320.

RUIZ Á.R. (2008), “El acceso al agua como factor de identificación de problemas de desarrollo agrario sostenible en el territorio del Alto Guadiana", Estudios Geográficos, 265, pp. 665-686.

RUNDSTROM R. \& KENZER M. (1989), “The decline of fieldwork in human geography”, The Professional Geographer, 41, pp. 294-303.

SANGUIN A. L. (1993), Vidal de La Blache (1845-1918). Un génie de la géographie, Paris, Belin, 383 p.

SORRE M. (1913), Les Pyrénées méditerranéennes : étude de géographie biologique, sous la direction de Paul Vidal de La Blache, Université de Paris, Armand Colin.

SANTOS M. (2000), La naturaleza del espacio. Técnica y Tiempo. Razón y Emoción, Madrid, Ariel, 348 p.

SAUER C. (1956), "The education of a geographer", $52^{\text {th }}$ meeting of the Annals of the Association of American Geographers, Montreal, 46, pp. 287-299.

SUNDBERG J. (2003), "Masculinist Epistemologies and the Politics of Fieldwork in Latin Americanist Geography", The Professional Geographer, 55, 2, pp. 180-190.

SWYNGEDOUW E. (1999), "Modernity and Hybridity: Regeneracionismo, the Production of Nature and the Spanish Waterscape, 1890-1930", Annals of the Association of American Geographers, 89, 3, pp. 443-465.

SWYNGEDOUW E. (2004), Social Power and the Urbanization of Water: Flows of Power, Oxford, Oxford University Press, $209 \mathrm{p}$.

SWYNGEDOUW E. (2007), “TechnoNatural Revolutions - the Scalar Politics of Franco's HydroSocial Dream for Spain 1939-1975", Transactions, Institute of British Geographers, 32, 1, pp. 9-28.

WOLFF D. (2006), “Albert Demangeon : un géographe face au monde rural (jusqu'en 1914)”, Ruralia [En ligne], 18/19.

WOLFF D. (2008), “Albert Demangeon : Un géographe moderne face au terrain”, Communication présentée au colloque À travers l'espace de la méthode : les dimensions du terrain en géographie, Arras, 18-20 juin 2008.

\section{NOTES}

1. Par exemple en 2001, la Geographical Review dans le numéro spécial nº 91 intitulé "Doing Fieldwork" propose une recompilation d'expériences et de réflexions sur le sujet. En 2010 la revue L'Information Géographique consacre un numéro (vol. 74) sur le terrain. Plus récemment, mentionnons le n687-688 (5-6/2012) des Annales de Géographie, intitulé “Terrains de je. (Du) sujet (au) géographique".

2. Le premier terrain, dans la région du haut Guadiana en Espagne (Communauté Autonome de Castilla-La Mancha), fut réalisé dans le cadre d'un mémoire de fin d'étude (2009-2010) à l'Université Libre de Bruxelles sous la direction du professeur Mr Vandermotten. Quant au deuxième, dans la province de Mendoza en Argentine il fut réalisé dans le cadre d'un mémoire de master de l'Université de Séville (2010-2011), sous la direction du professeur Leandro Ituarte del Moral et financé par une bourse octroyée par cette université.

3. L'irrigation en Méditérranée pour Jean Brunhes (1902), la Picardie pour Albert Demangeon (1905), la Flandre pour Raoul Blanchard (1906), les Pyrénées pour Max Sorre (1913), ... 
4. Comme d'autres auteurs l'ont souligné (Blanchon \& Graefe, 2012; Benjaminsen \& Svarstad, 2009; Molle, 2012) nous préférons garder ici le terme anglais, afin de la distinguer de l'écologie politique.

5. D'après l'expression "Putting thing in fluid terms" (Linton, 2011). Il est curieux d'observer que cette tendance à recourir à ces métaphores de circulation, de flux voire de processus fluides avait déjà été mise en avant par Jean Gottmann fin des années 1940 quand il défendait que les AngloSaxons étaient plus sensibles que les géographes français au caractère dynamique des faits de géographie humaine (Gottmann, 1947).

6. Ce processus qui impliquait le passage des terres des mains de la noblesse, de l'église, des ordres militaires et municipaux aux mains d'une minorité, la haute bourgeoisie, n'a cependant pas altéré fondamentalement la structure de la propriété.

7. Paco la grenouille était le surnom donné au dictateur Francisco Franco pour sa manie de construire des barrages.

8. Une loi des eaux obsolète et déficiente, couplée à l'initiative privée soutenue par l'État puis par la PAC à partir de 1986 selon les conjonctures économiques qu'offraient certaines cultures sur le marché mondial, sont les principales causes de la surexploitation des aquifères manchego.

9. La "Nouvelle vitiviniculture" est devenue un secteur particulièrement prisé pour le capital étranger.

10. Revue Belge de Géographie, "Hommage à Pierre Gourou (1900-1999), 1998, p. 196.

11. En ciblant particulièrement les infrastructures et techniques d'irrigation, et les systèmes de cultures et les sources d'alimentation en eau (superficielles ou souterraines) auxquelles elles étaient associées.

\section{RÉSUMÉS}

Les géographes ont peu théorisé les pratiques du travail de terrain mais de récentes publications ont montré que ce dernier pouvait encore constituer une entrée judicieuse pour le géographe, à condition de l'exposer à la lumière de certaines réflexions épistémologiques en sciences sociales. Ce nouvel intérêt pour le terrain semble cependant se distancier des expériences et apports accumulés en matière de terrain par certains géographes classiques ou "modernes". Cet article soutient que, parmi les gestes de métiers de ces derniers, certaines techniques permettant d'encadrer l'observation directe ou sensorielle méritent d'être reconsidérées afin de souligner leurs rôles éminemment pédagogiques dans la formation du géographe. Il expose donc la manière dont l'auteur s'est penché sur l'histoire et l'actualité du terrain en géographie pour alimenter sa pratique dans le cadre de deux études portant sur la gestion de l'eau en milieu rural dans le sud de l'Espagne et l'ouest de l'Argentine.

Geographers are yet to theorize much about the politics and practices of fieldwork. Nevertheless, recent publications have shown that this practice could still constitute a judicious approach in geography as long as it was exposed to new epistemological thoughts in social science. These new considerations seem, however, to differentiate themselves from the accumulated experiences and contributions in the field by some modern geographers. This paper considers that, among the professional practice of the aforementioned authors, some techniques used to manage direct or sensory observation should be revised with the aim of underlining their educational role. It exposes how the author explored the history and recent practice of fieldwork to feed his own 
investigation constructed from two personal experiences in rural Southern Spain and in Western Argentina.

INDEX

Mots-clés : travail de terrain, paysage, irrigation, Castilla-La Mancha, Mendoza

Keywords : fieldwork, landscape

\section{AUTEUR}

\section{ROBIN LARSIMONT}

Université de Buenos Aires (UBA), doctorant, Instituto de Ciencias Humanas, Sociales y Ambientales (INCIHUSA-CONICET) de Mendoza, doctorant, rlarsimont@mendoza-conicet.gob.ar 